\title{
Analysis of Damage Mechanism of Tunnel Lining Structure under the Coupling Action of Active Fault
}

\author{
Guotao Yang $\mathbb{D}^{1},{ }^{1}$ Sujian $M a,{ }^{2}$ Liang Zhang $\mathbb{D}^{2},{ }^{2}$ Xinrong Tan, ${ }^{3}$ Rui Tang, ${ }^{4}$ and Yang Liu ${ }^{5}$ \\ ${ }^{1}$ China State Railway Group Co., Ltd., Beijing 100844, China \\ ${ }^{2}$ Department of Civil Engineering, Southwest Jiaotong University, Chengdu 610031, China \\ ${ }^{3}$ China Railway Eryuan Engineering Group Co., Ltd., Chengdu 610031, China \\ ${ }^{4}$ Sichuan Highway Planning, Survey, Design and Research Institute Ltd., Chengdu 610041, China \\ ${ }^{5}$ Zhongke (Hunan) Advanced Rail Transit Research Institute Co., Ltd., Zhuzhou 412000, China \\ Correspondence should be addressed to Liang Zhang; zhangliangdpme@my.swjtu.edu.cn
}

Received 8 March 2021; Accepted 6 May 2021; Published 20 May 2021

Academic Editor: Guang-Liang Feng

Copyright (C) 2021 Guotao Yang et al. This is an open access article distributed under the Creative Commons Attribution License, which permits unrestricted use, distribution, and reproduction in any medium, provided the original work is properly cited.

\begin{abstract}
To reveal the failure mechanism of tunnel structure under active fault movement, based on the pseudostatic elastoplastic finite element method, the failure modes of the tunnel lining are studied under different movement ratios of strike-slip faults and thrust faults with $45^{\circ}$ dip angle by using numerical simulation. The results show that the range of significant lining failure section can be determined according to any direction of the coupling fault movement decomposition direction, and the damage effect is determined by the overall movement amount of the coupling fault. The significant damage area of the lining under the action of the coupling fault is the same as the area of deformation, which mainly manifests as tensile failure. Compressive failure occurs in the boundary area between the fracture zone and the hanging wall and foot wall. The plastic strain is the largest in the area where the arch waist and the arch bottom intersect. The development of tunnel lining plastic zone under coupling fault is from arch top and arch bottom to both sides of the arch waist. The development of the plastic zone under active fault is mainly determined by the form of fault with a large ratio. The research results can provide a reference for the design and safety evaluation of tunnel crossing active faults.
\end{abstract}

\section{Introduction}

The underground structure has better antivibration performance under the pressure of surrounding rock, but related studies have shown [1-4] that the tunnel structure that crosses an active fault will cause more catastrophic damage under the movement of the active fault and even cause the structure to collapse [5-7], which will cause considerable economic losses. In the construction of tunnel engineering, relevant codes stipulate the fault section based on the principle of avoidance. However, the direction of the tunnel route nowadays is more inclined to its demand for traffic function, resulting in increasing section tunnels inevitably crossing the active fault zone $[8,9]$.

At present, domestic and foreign experts and scholars have achieved some meaningful results in the study of tunnels through active faults. Chen [10] established the surface fracture zone length caused by fault movement in the Taiwan area, the east and west of China, Japan, and India. Two statistical regression formulas for fracture length and magnitude were established to estimate the length of the surface fracture zone through the magnitude and estimate the magnitude through the length of the surface fracture. Wells and Coppersmith [11] collected 244 relatively complete seismic records for statistical analysis and established the relationship between the magnitude and surface, underground fracture length, and surface movement worldwide. Cole and Lade [12] gave a simplified model for predicting the shape and distribution of ground fractures caused by bedrock movement through sand box test and theoretical analysis and summarized three key factors that have an impact: soil thickness, dilatancy angle, and fault dip. 
Bransby et al. $[13,14]$ simulated normal and inverse faults with a fault dip of $60^{\circ}$ through centrifuge tests, considering the effect of changing the load on the upper part of the soil layer and changing the position of the rigid foundation on the fracture of the soil layer. Lin et al. $[15,17]$ simulated the deformation and failure characteristics of tunnels under thrust fault movement through the designed fault test device, studied the influence of relevant parameters on the tunnel structure, and verified the experimental conclusions by numerical simulation. Sabagh and Ghalandarzadeh [18] used a series of finite element numerical models to evaluate the behavior of tunnel and reverse fault intersections. The numerical simulation results of $60^{\circ}$ reverse fault in free field mode and tunnel mode are verified by centrifugal test. An et al. [19] proposed a two-level design method of fault dislocation and established a three-dimensional finite element model to estimate the tunnel response under fault dislocation. Zhang et al. [20] established a three-dimensional geomechanical model with faults, used the regional inversion method to obtain the regional stress field distribution under the fault condition, and used the mathematical statistics method to obtain the initial stress field of Daxiangling tunnel. Taking the inclined shaft of Muzhailing highway tunnel as the engineering background, Tao et al. [21] evaluate the supporting effect of carbonaceous slate and the deformation of surrounding rock through model test.

The above research results are of great significance to the construction of tunnels crossing active faults, but also face some complex problems. For example, the preliminary work of statistical data is complicated, and the data is numerous, the mechanism of fault fracture is more complicated, the dispersion of test results is large, the numerical simulation has limitations, etc. Current research needs to be further in depth for guiding engineering practice and seismic design. On the basis of summarizing previous studies, this paper uses a pseudostatic elastic-plastic finite element analysis method based on a domestic tunnel project and estimates the structural damage of the lining from the deformation mechanism, the maximum principal stress distribution and the development of the plastic zone through a large number of calculations, and the failure mechanism of the lining under the complex coupling action of the fault is revealed.

\section{Three-Dimensional Finite Element Numerical Model Establishment and Parameter Selection}

Relying on an actual tunnel in China, based on the threedimensional finite element software ABAQUS, this paper established a three-dimensional finite element model of the fracture zone with a $45^{\circ}$ dip angle with different strike-slip and thrust ratios. According to the survey data in the preconstruction period, the width of the model fracture zone is $40 \mathrm{~m}$ and $180 \mathrm{~m}$ before and after the fracture zone is taken to establish a tunnel calculation model for coupling active fault. The cross-sectional shape of the tunnel is drawn with a five-centered circle similar to the actual project. The maximum inner diameter is $10 \mathrm{~m}$, the height is $7 \mathrm{~m}$, the lining wall thickness is $0.5 \mathrm{~m}$, and the tunnel buried depth is $28 \mathrm{~m}$. The domestic tunnel construction empirical practice believes that when the transverse section size is 5-10 times the tunnel section size, the artificial boundary has little effect on the calculation results [22]. Therefore, the cross section size of the model is $70 \mathrm{~m}$, which is 10 times the height of the tunnel and 7 times the width. The model size is $400 \mathrm{~m} \times 70 \mathrm{~m} \times 70 \mathrm{~m}$, and the model calculation diagram is shown in Figure 1.

As shown in Figure 1, the abcd segment is the hanging wall area of the active fault, and the efgh segment is the foot wall area of the active fault. The fault movement is realized by applying constraint boundary conditions and applying displacement load boundary conditions. This calculation model simulates the failure of the lining under different fault displacements and different ratios of the two displacements. The coupling fault displacement is dominated by thrust faults, and the maximum displacement is $4.0 \mathrm{~m}$. Considering the most unfavorable effects, the calculation model sets a lower movement rate, and each analysis step movement is $0.1 \mathrm{~m}$; that is, the simulated movement rate is $0.1 \mathrm{~m} / \mathrm{s}$, which gives the surrounding rock sufficient deformation and maximizes the damage of the tunnel lining $[15,16,23]$. To explore the damage mechanism of the tunnel lining structure under the coupling action of active faults, the constitutive relationship of the lining in the calculation model adopts the concrete structure damage constitutive, the lining material is C55 concrete, and the stress-strain relationship is defined in accordance with the code [24], as is shown in Table 1. The hanging wall and foot walls of the active fault and the fracture zone adopt the Mohr-Coulomb constitutive relationship. The reason for the fault movement along the slip surface of the fracture zone is that the fracture zone has low strength and is easy to damage. Therefore, the calculation model appropriately reduces the strength of the fracture zone according to a safety factor of 0.8 . The specific calculation parameters are shown in Table 2. The model design considers the material properties and differences between the lining and the surrounding rock and defines the contact between the two as friction, and the friction parameter is 0.4 [25].

The model calculation is carried out in three steps. The initial ground stress is applied to the unexcavated rock and soil, so that it will not deform under the action of gravity, which simulates the rock and soil under long-term consolidation. After the initial ground stress is balanced, the tunnel excavation and the construction of the lining are simulated. The surrounding rock pressures all act on the lining and the lining will no longer be disturbed by deformation. After the lining and surrounding rock reach the state of balance, displacement loads are applied to the strikeslip direction ( $Y$-direction) and thrust direction ( $Z$-direction) of the hanging wall of the active fault to realize the coupling and movement of the active fault. The boundary conditions of the foot wall remain unchanged, and the load boundary conditions are shown in Figure 2.

Both the soil element and the lining element of the calculation model adopt hexahedral elements. The mesh is densely divided into the fracture zone and the rock mass 


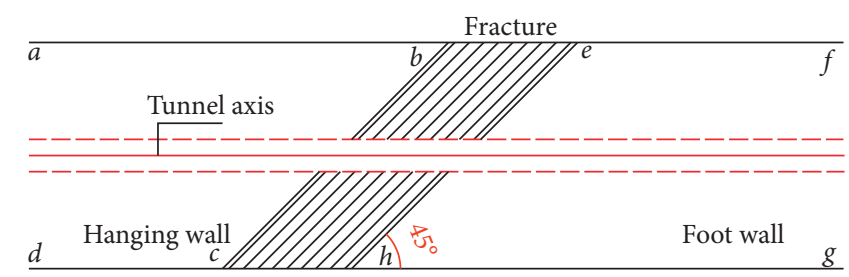

Figure 1: Schematic diagram of the calculation model for dip angle $45^{\circ}$.

TABLE 1: Material parameters of tunnel lining.

\begin{tabular}{lccccc}
\hline Model & Density $\left(\mathrm{kg} / \mathrm{m}^{3}\right)$ & Elastic modulus $(\mathrm{MPa})$ & Poisson's ratio & Compressive yield stress $(\mathrm{MPa})$ & Tensile yield stress $(\mathrm{MPa})$ \\
\hline Lining & 2400 & $2.648 \times 10^{4}$ & 0.167 & 32.5 & 2.64 \\
\hline
\end{tabular}

TABle 2: Material parameters of surrounding rock and fault.

\begin{tabular}{lccccc}
\hline Model & Density $\left(\mathrm{kg} / \mathrm{m}^{3}\right)$ & Elastic modulus $(\mathrm{MPa})$ & Poisson's ratio & Internal friction angle $\left(^{\circ}\right)$ & Cohesion $(\mathrm{kPa})$ \\
\hline Fault & 2300 & $1.6 \times 10^{3}$ & 0.4 & 28 & $0.1 \times 10^{3}$ \\
Surrounding rock & 2400 & $3.5 \times 10^{3}$ & 0.32 & 35 & $0.4 \times 10^{3}$ \\
\hline
\end{tabular}

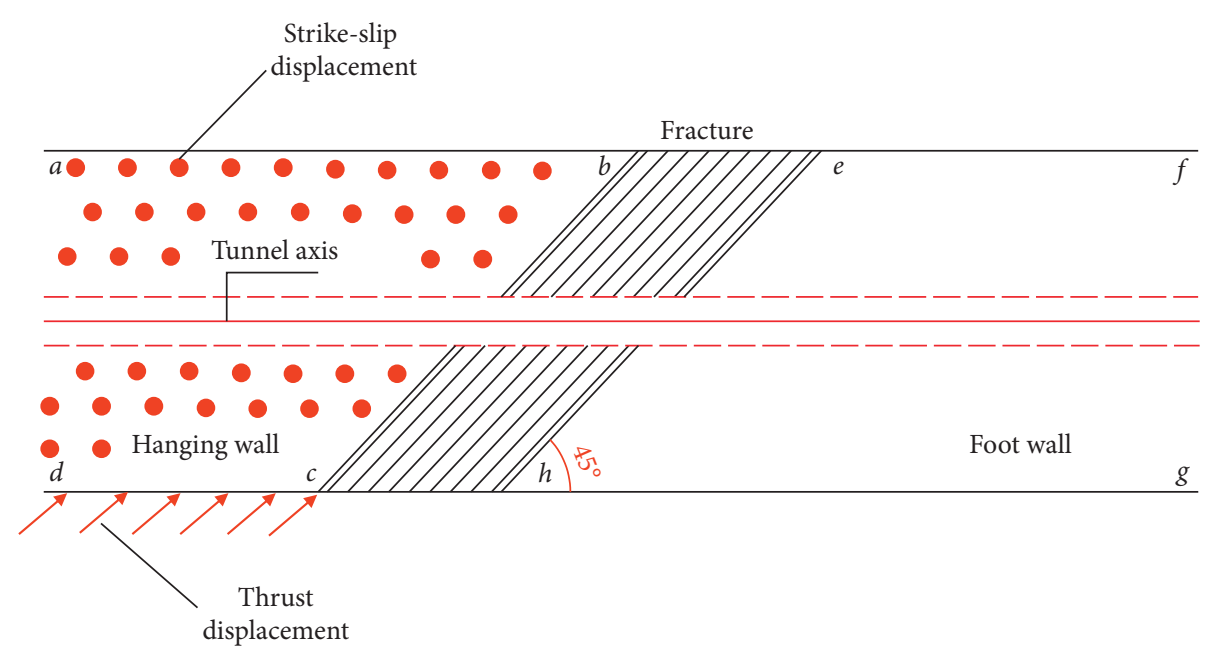

Figure 2: Schematic diagram of the coupling fault displacement load distribution.

around the tunnel lining, and the soil mesh is sparse near the boundary, which ensures the accuracy and reliability of the calculation results and the efficiency of the calculation. The mesh is shown in Figure 3.

\section{Safety Analysis of Tunnel Lining with Different Movement Amounts under the Coupling Action of Active Faults}

Under strike-slip fault movement, the hanging wall and foot wall move horizontally along the fracture surface. The stress source of fault movement is the shearing action on both sides. The movement surface is smooth, and the shearing effect is obvious [26]. Thrust faults are forward faults with a large displacement distance, showing strong compression [27]. Because the fault movement of the calculation model is dominated by thrust faults, this paper selects the tunnel lining arch vault measurement points under different movements with the ratio of strike-slip fault to thrust fault at $1: 2$ for safety analysis.

Fault movement is divided into stick-slip movement and creeping movement. Stick-slip movement is a kind of rapid fracture motion, and creeping movement is a slow movement that occurs over time. Studies have shown that the slow movement of the fault gives the soil a sufficient deformation process, and the failure form is larger than the stick-slip movement [28]. To explore the influence of the movement amount on the tunnel lining under the coupling action of active faults, this paper takes the lining response when the continuous movement amount of the thrust fault in the coupling fault is $0.1 \mathrm{~m}, 0.2 \mathrm{~m}, 0.3 \mathrm{~m}, 0.4 \mathrm{~m}, 0.5 \mathrm{~m}$, and $0.6 \mathrm{~m}$. And the failure mechanism of the tunnel lining under different displacements of the coupling fault is revealed through the longitudinal displacement distribution of the lining along the tunnel, the distribution of the maximum 

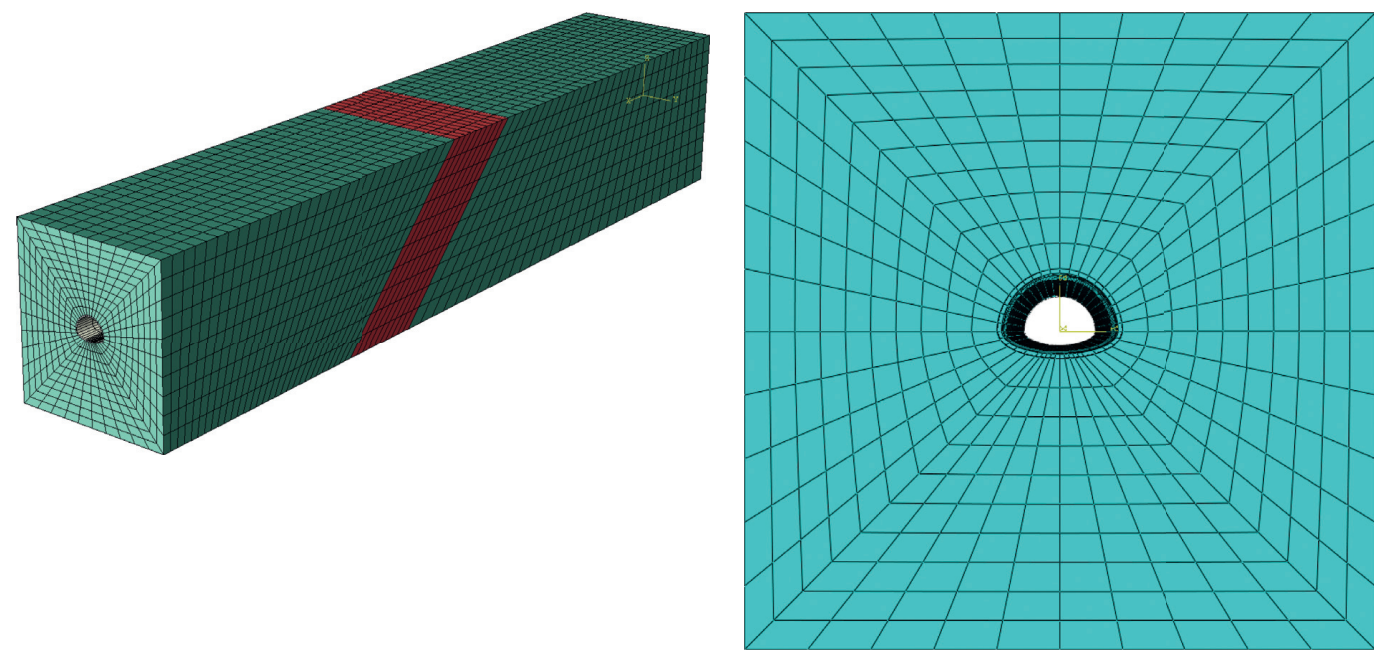

(a)

(b)

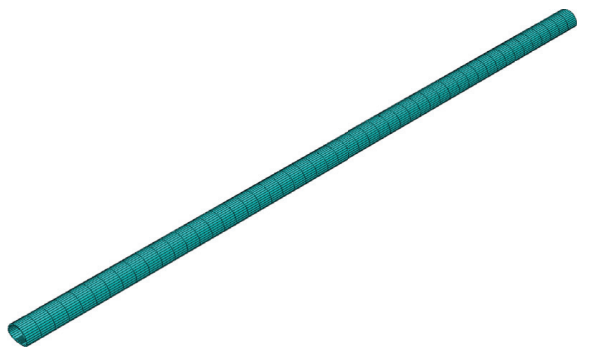

(c)

Figure 3: The meshing diagram of calculation model. (a) The meshing diagram of the overall calculation model. (b) The meshing diagram of the transverse section of the calculation model. (c) The meshing diagram of tunnel lining.

principal stress, and the distribution of the development range of the plastic zone.

\subsection{Displacement Response Analysis of Tunnel Lining with} Different Movement Amounts under the Coupling Active Fault. Since the coupling fault displacement load is applied in the $Y$-direction and the $Z$-direction, the $Y$-direction and $Z$ direction tunnel lining vault displacement distribution is taken for analysis. Figure 4(a) is the deformation cloud diagram of lining when the fault displacement is $0.4 \mathrm{~m}$, and Figure $4(\mathrm{~b})$ shows the $Y$-direction displacement distribution curve of the lining under different displacements when the coupling ratio between strike-slip fault and thrust fault is $1: 2$, and Figure 4(c) is the $Z$-direction displacement distribution curve of the lining.

Comprehensive analysis of Figure 4 shows that the coupling fault movement drives the lining in the hanging wall area to move in the same form in the strike-slip direction and the thrust direction. The movement platform appears along the lining displacement curve in the longitudinal direction of the tunnel, and the range of the platform is roughly the same as that of the hanging wall of the fault. The coupling fault is $1: 2$ moving in the $Y$ - and $Z$-directions, and the lining displacement response is roughly $1: 2$, and the projection of the lining displacement value in the two directions is consistent with the displacement load value. The shape of the lining displacement curve in two directions under the coupling fault movement is " $\mathrm{S}$ "-shaped distribution, and the deformation shows a steep drop in the projection area at the bottom of the fracture zone along the longitudinal direction. Since the dip angle of the fracture zone is $45^{\circ}$, the fault projection area is within the range of $110 \mathrm{~m} \sim 220 \mathrm{~m}$. With the increase of the movement amount of the active fault, the curved platform continues to rise, the slope of the curve in the steep decline section gradually increases, but the range and location of the platform does not change significantly. The steep drop section of the $Y$-direction curve is roughly within the range of $140 \sim 200 \mathrm{~m}$, and the steep drop section of the $Z$-direction curve is roughly within the range of $140 \sim 200 \mathrm{~m}$. Therefore, the significant damage area of the lining under the coupling active fault is roughly located in the fracture zone. With the increase of the movement of the active fault, the damage range does not change significantly, but the damage effect increased significantly. The significant deformation section of the lining can be judged according to the displacement curve of the projection direction of the coupling fault motion.

3.2. Stress Response Analysis of Tunnel Lining with Different Movement Amounts under the Coupling Active Fault. Since the tunnel lining material is C55 concrete, the maximum principal stress is used as the lining stress response analysis index to study the structural damage mechanism of 

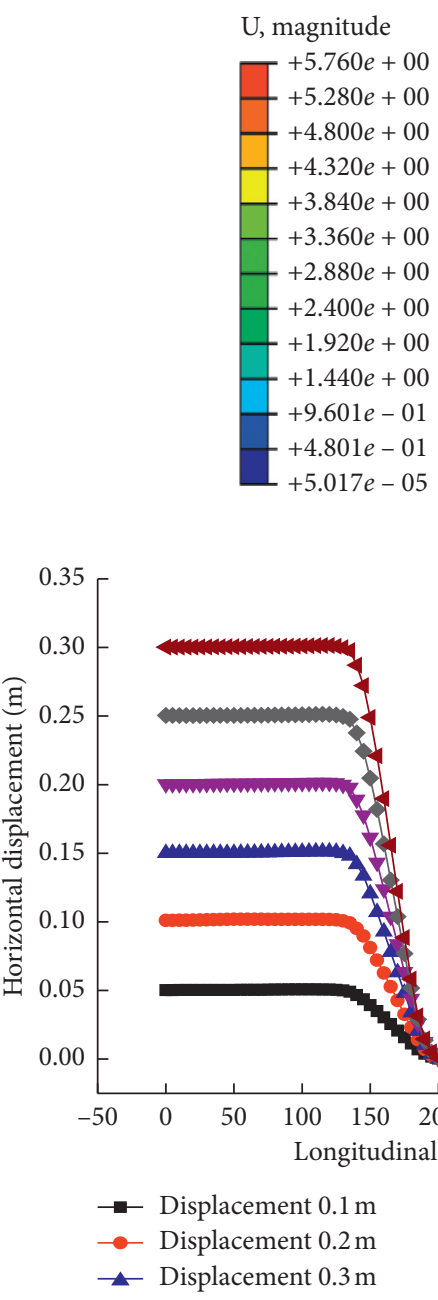

(b)

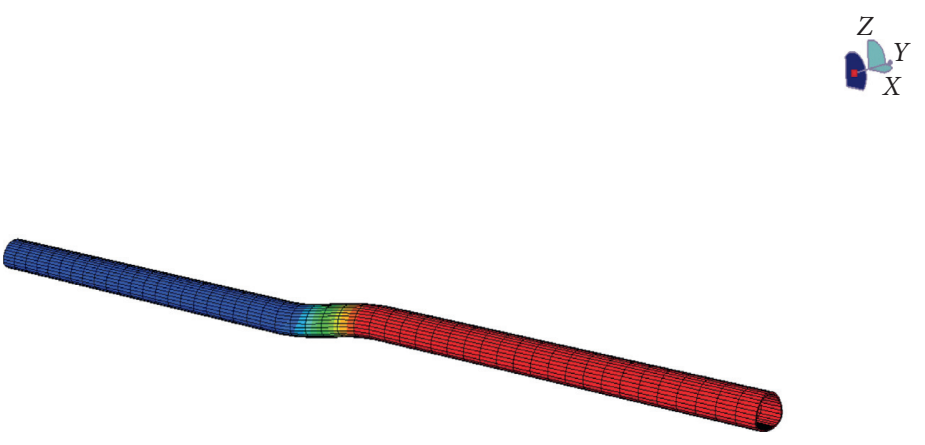

(a)

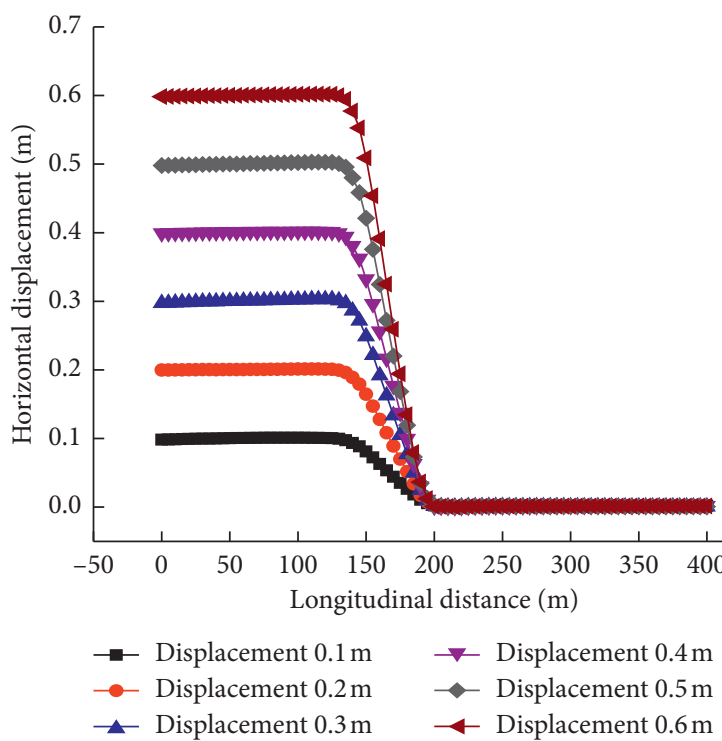

(c)

Figure 4: Displacement diagram in different directions under coupling fault. (a) The deformation cloud diagram of lining when the fault displacement is $0.4 \mathrm{~m}$. (b) $Y$-direction displacement curve of lining with different movement amounts in the coupling ratio of $1: 2$. (c) Z-direction displacement curve of lining with different movement amounts in the coupling ratio of $1: 2$.

the lining. According to the calculation results, the maximum principal stress distribution curve of the lining with different movements under the coupling fault action is shown in Figure 5.

From a comprehensive analysis of Figure 5, it can be seen that the maximum principal stress curve of the lining under the dip angle of $45^{\circ}$ coupling fault movement is symmetrically distributed along the longitudinal direction of the tunnel with the axis of symmetry approximately $140 \mathrm{~m}$. The shape of the distribution curve of the maximum principal stress of the lining under different displacements is roughly similar. When the thrust direction of the coupling fault is misaligned by $0.1 \mathrm{~m}$, the maximum principal stress of the hanging wall is larger than other displacements. The maximum principal stress is the tensile stress before $140 \mathrm{~m}$ along the longitudinal direction of the tunnel, and the stress value gradually increases with the longitudinal distance. The maximum tensile stress appears near $140 \mathrm{~m}$, and the compressive stress appears near $200 \mathrm{~m}$ in the longitudinal direction of the tunnel, and the lining begins to undergo compression failure. The maximum principal stress of the lining in the foot wall area is basically 0 . The foot wall lining of the coupling fault movement shows obvious tensile failure, and the significant increase area of the maximum principal stress is located at the location of the fracture zone. Compression failure occurs at the contact position of the foot wall and the fracture zone, and the tensile failure range of the lining is roughly consistent with the position of the steep drop of the lining displacement curve. Therefore, it can be inferred that the significant damage area of the lining under the action of the coupling fault is the same as the significant area of displacement deformation, which is roughly located near the fracture zone and mainly manifests as tensile failure.

3.3. Analysis of the Development of the Plastic Zone of the Tunnel Lining under the Coupling Effect of Active Faults with Different Displacements. The real-time monitoring method of tunnel lining and the analysis and processing of deformation data have important practical significance for ensuring the stability of the tunnel [29-31]. As one of the important 


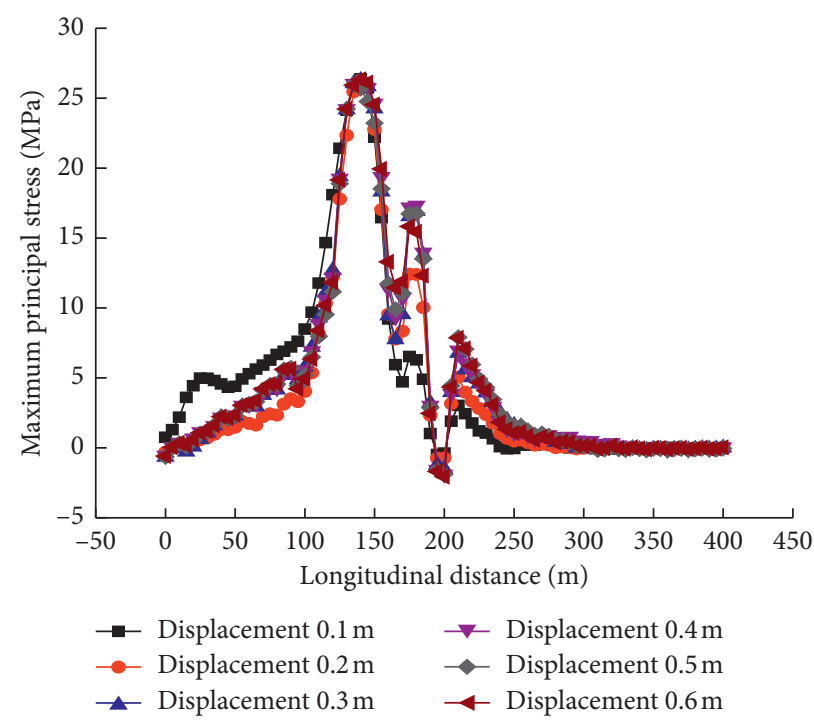

Figure 5: Maximum principal stress distribution curve of the lining with different displacements under coupling fault action.

indicators of lining structure damage, the plastic zone plays an essential role in the monitoring of tunnel construction. Figure 6 is the cloud diagram of the development of the plastic zone of tunnel lining with different displacements under the coupling fault action.

Comprehensive analysis of Figure 6 shows that, at the beginning of the coupling fault, the plastic zone is distributed on the lining vault and the arch bottom, and the distribution range is roughly concentrated on the vault at the intersection of the hanging wall and the fracture zone and the arch bottom at the intersection of the foot wall and the fracture zone. As the amount of coupling fault movement increases, the plastic zone at the top and bottom of the arch continues to expand and connect with both sides of the arch waist. When the coupling fault is displaced for a certain distance, the position of the plastic zone of the tunnel lining changes from the top and bottom of the arch to both sides of the arch waist. The development range of the plastic zone is concentrated in the fracture zone, and the plastic zone on both sides of the arch waist increases with the increase of the active fault movement amount. The cloud diagram of the development of the plastic zone scatters from the center to the surroundings in a discord shape, and the plastic strain is the largest in the area where the arch waist and the arch bottom intersect. The development law of the plastic zone of tunnel lining under coupling fault movement is similar to that of tunnel lining under thrust fault movement. Therefore, the development of plastic zone under active fault movement is mainly determined by the form of fault movement with larger ratio.

\section{Safety Analysis of Tunnel Lining with Different Displacement Ratios under the Coupling Action of Active Faults}

To explore the influence of different movement ratios on the tunnel lining under the coupling action of active faults, three different ratios of strike-slip and thrust movement ratios of
$1: 1,1: 2$, and $1: 3$ are taken in this paper, to study the response of the lining when the continuous displacement of the thrust fault is $1.4 \mathrm{~m}$ in the coupling fault. And the failure mechanism of the tunnel lining under the different displacements of the coupling fault is revealed through the longitudinal displacement distribution of the lining along the tunnel, the distribution of the maximum principal stress, and the distribution of the development range of the plastic zone.

4.1. Displacement Response Analysis of Tunnel Lining with Different Movement Ratios under the Coupling Active Fault. Coupling active faults are a combination of strike-slip faults and thrust faults. Therefore, it is necessary to study the $Y$ and $Z$-direction lining displacement curves separately. The ratio of strike-slip movement and thrust movement of the $45^{\circ}$ fracture zone is $1: 1,1: 2$, and $1: 3$, as shown in Figure 7.

It can be seen from Figure 7 (a) that since the movement of the active fault is dominated by the thrust fault, the data of different movement ratios of the strike-slip fault and the thrust fault under the coupling fault under $1.4 \mathrm{~m}$ of the thrust fault movement are selected for analysis. Therefore, the $Y$-direction lining displacement curve has a movement platform, and the range of the platform is roughly the same as the bottom of the fault hanging wall. With the change of the coupling fault movement ratio, the platform height of the $Y$-direction lining displacement curve gradually decreases, and the platform range does not change significantly. The lining displacement value is roughly the same as the $Y$-direction displacement load applied value, the lining and the hanging wall do the same form of movement. The Y-direction lining displacement curve is distributed in an " $S$ " shape, and there is a steep drop in the projection area at the bottom of the fracture zone along the longitudinal direction. With the increase of the movement ratio, the dip angle of the steep-descent section increases continuously, and the damage effect increases significantly, while the significant 


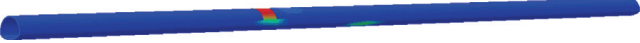

(a)

(c)

(e) (b)

(d)

(f)

Figure 6: The cloud diagram of the development range of the plastic zone of tunnel lining with different displacements under the coupling fault action. (a) The cloud diagram of plastic zone of $0.1 \mathrm{~m}$ tunnel lining with coupling fault movement. (b) The cloud diagram of plastic zone of $0.2 \mathrm{~m}$ tunnel lining with coupling fault movement. (c) The cloud diagram of plastic zone of $0.3 \mathrm{~m}$ tunnel lining with coupling fault movement. (d) The cloud diagram of plastic zone of $0.4 \mathrm{~m}$ tunnel lining with coupling fault movement. (e) The cloud diagram of plastic zone of $0.5 \mathrm{~m}$ tunnel lining with coupling fault movement. (f) The cloud diagram of plastic zone of $0.6 \mathrm{~m}$ tunnel lining with coupling fault movement.

damage range is roughly concentrated in the same range of the fracture zone.

It can be seen from Figure $7(\mathrm{~b})$ that the coupling fault $Z$ direction bears a vertical displacement load of $1.4 \mathrm{~m}$, and the lining displacement curve shows a movement platform along the longitudinal direction of the tunnel. The range of the platform is the same as the bottom of the hanging wall of the fault, and the displacement curve shows an "S"-shaped distribution of the same shape. The $Z$-direction displacement curve shows a steep drop in the fracture zone along the longitudinal direction of the tunnel, and the significant damage area of the lining at $45^{\circ}$ dip angle of the fracture zone is approximately $140 \mathrm{~m} \sim 200 \mathrm{~m}$. Therefore, the significant damage area of the lining under the coupling active fault movement is roughly located in the fracture zone, and the two directions are the same. With the increase of the movement ratio, the damage effect becomes more obvious while the damage range is basically unchanged. The range of the significant damage section of the lining can be determined according to any direction of the coupling fault movement decomposition direction, and the damage effect needs to be determined by the overall movement of the coupling fault.

4.2. Response Analysis of Tunnel Lining Stress with Different Movement Ratios under the Coupling Active Fault. To explore the influence of the stress distribution on the tunnel lining under different movement ratios of strike-slip and thrust faults, the maximum principal stress distribution along the longitudinal length of the tunnel is calculated according to the calculation results, as shown in Figure 8.

Comprehensive analysis of Figure 8 shows that the maximum principal stress curve of the lining under different movement ratios of the $45^{\circ}$ dip angle coupling fault is symmetrically distributed along the longitudinal direction of the tunnel with the axis of symmetry approximately $140 \mathrm{~m}$. The shape of the maximum principal stress distribution curve of the lining under different displacement ratios is roughly similar, and the maximum principal stress distribution curve shows a trend of first increasing and then decreasing as a whole. The stress increase area along the longitudinal length of the tunnel is roughly located near the fracture zone. The maximum principal stress continues to increase before $140 \mathrm{~m}$, and the maximum appears at $140 \mathrm{~m}$, which is a tensile stress, and the lining shows tensile failure. Compressive stress occurs in the lining at the boundary between the fracture zone and the hanging wall and foot wall, and compression failure occurs. The maximum principal stress of the lining in the foot wall is basically zero. The lining tensile stress distribution area under the coupling fault movement is wide, so the lining mainly shows tensile failure. The significant tensile failure range of the lining is roughly consistent with the position of the steep drop of the lining displacement curve, showing a consistent change law.

4.3. Analysis of the Development of the Plastic Zone of the Tunnel Lining under the Coupling Effect of Active Faults with Different Movement Ratios. In order to explore the influence of the development of the plastic zone on the tunnel lining under different movement ratios of the coupling active fault, according to the calculation results, the cloud diagram of the development range of the plastic zone of the lining under different movement ratios when the coupling fault is displaced at a $45^{\circ}$ angle of $1.4 \mathrm{~m}$ is obtained, as shown in Figure 9.

Comprehensive analysis of Figure 9 shows that the distribution of the plastic zone under different ratios of the coupling faults is roughly concentrated in the middle of the fracture zone. When the coupling fault movement ratio is 1 : 1 , the plastic zone is concentrated in the arch bottom and waist area of the tunnel lining, and the arch bottom is mainly distributed, and the plastic strain value is discord shape and scatters to the surroundings. With the increase of the movement ratio, the position of the plastic zone of the lining begins to expand from the arch bottom to both sides of the arch waist, and the range increases accordingly. The development law of the plastic zone area under different proportions of movement is similar to the development law of tunnel lining under thrust fault. Therefore, it can be inferred that the development of lining plastic zone is related to the fault movement formed in the dominant direction in the coupling fault, and the development law is affected by the development law of the lining plastic zone under its sole action. The lining plastic zone is roughly concentrated in the fracture zone, which is consistent with the tensile stress 


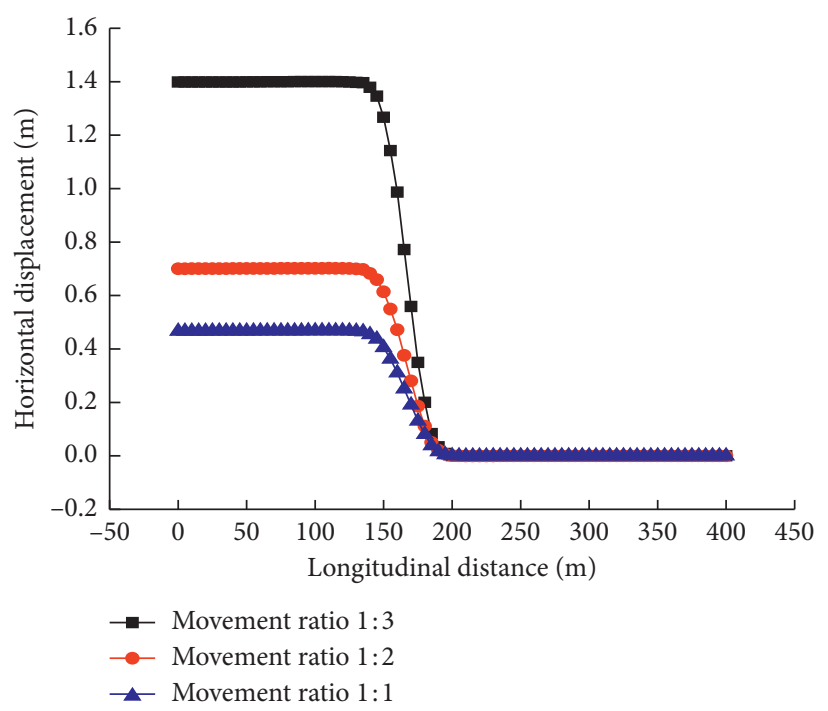

(a)

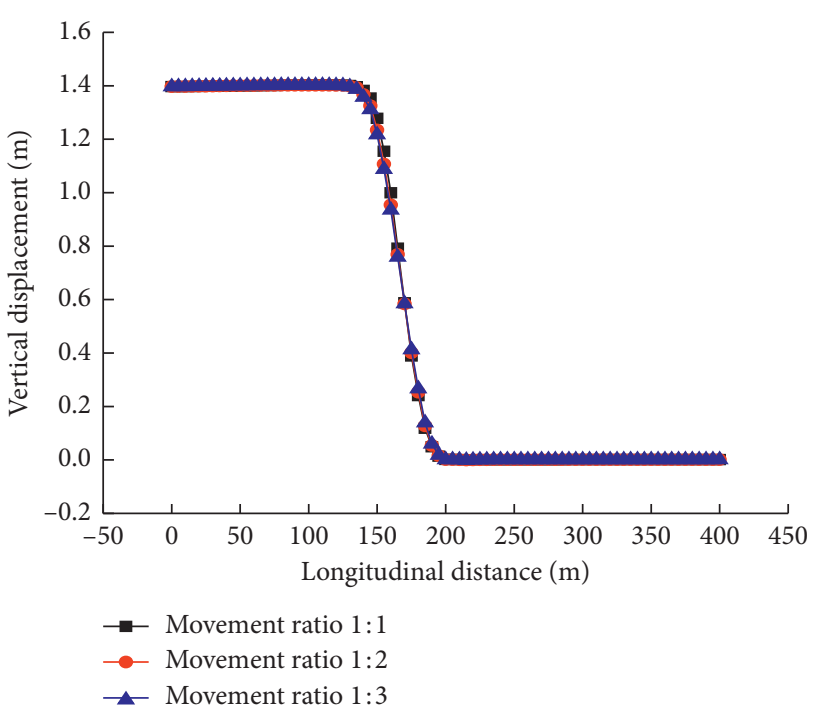

(b)

FIgURE 7: Displacement curves of tunnel lining with different movement ratios of $45^{\circ}$ dip angle under coupling fault. (a) $Y$-direction lining displacement curve with different movement ratios under coupling fault. (b) $Z$-direction lining displacement curve with different movement ratios under coupling fault.

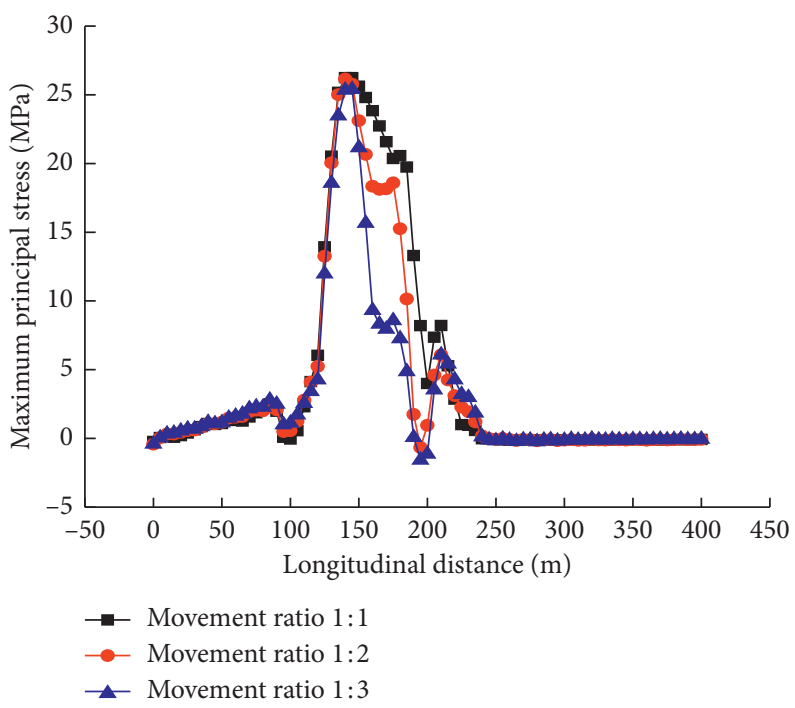

FIGURE 8: Maximum principal stress distribution curve of the lining with different movement ratios under the coupling fault.

(a)

(b)

(c)

Figure 9: The cloud diagram development range of the plastic zone of the lining with different ratios of $1.4 \mathrm{~m}$ at the $45^{\circ}$ dip angle. (a) The cloud diagram of plastic zone of $1.4 \mathrm{~m}$ tunnel lining with a ratio of $1: 1$. (b) The cloud diagram of plastic zone of $1.4 \mathrm{~m}$ tunnel lining with a ratio of $1: 2$. (c) The cloud diagram of plastic zone of $1.4 \mathrm{~m}$ tunnel lining with a ratio of $1: 3$. 
distribution area of the maximum principal stress of the lining along the longitudinal direction of the tunnel.

\section{Conclusion}

This paper establishes a three-dimensional finite element tunnel model under the coupling movement of strike-slip and thrust faults, discusses the displacement, stress distribution, and plastic zone development of the tunnel lining under different movements and different movement ratios of the coupling faults, and reveals the damage mechanism of the lining under the active fault movement, and the conclusions are as follows:

(1) The coupling fault movement drives the lining in the hanging wall area to move in the same form in the strike-slip direction and the thrust direction. The significant damage area of the lining under the coupling active fault movement is roughly located in the fracture zone. With the increase of the movement amount of the active fault, the damage effect increases significantly. The range of the significant damage section of the lining can be determined according to any direction of the coupling fault movement decomposition direction, and the damage effect needs to be determined by the overall movement of the coupling fault.

(2) The tunnel lining of the coupling fault movement shows obvious tensile failure. The significant growth area of the maximum principal stress is located at the fracture zone, and compression failure occurs at the contact position of the foot wall and the fracture zone. The lining mainly shows tensile failure, and the significant tensile damage range of the lining is roughly consistent with the position of the steep drop of the lining displacement curve. It can be inferred that the significant damage area of the lining under the action of the coupling fault is the same as the significant displacement change area, which are roughly located near the fault zone and mainly manifested as tensile failure.

(3) At the beginning of the coupling fault, the plastic zone is distributed on the lining vault and the arch bottom. With the increase in the amount of coupling fault movement, the plastic zone at the top and bottom of the arch continues to expand and connect on both sides of the arch waist. The plastic strain is the largest in the area where the arch waist and the arch bottom intersect. The development of plastic zone under active fault movement is mainly determined by the form of fault movement with larger ratio.

\section{Data Availability}

The data used to support the findings of this study are available from the corresponding author upon request.

\section{Conflicts of Interest}

The authors declare that they have no conflicts of interest regarding the publication of this paper.

\section{Acknowledgments}

This study was supported in part by the Science and Technology Research and Development Plan of China National Railway Corporation Limited (no. K2019G009); National Natural Science Fund Project (no. 52078426); National Key Research and Development Plan (no. 2018YFE0207100); Sichuan Provincial Science and Technology Support Project (nos. 2020YJ0253, 2020YFSY0060, 2019JDRC0133, and 2019JDRC0134); China Railway Eryuan Engineering Group Co., Ltd. Scientific Research Project (KYY2019034(19-22) and KYY2019070(19-20)); and China National Railway Group Co., Ltd. Scientific Research Project (nos. SY2016G003 and N2020T004).

\section{References}

[1] P. Geng, C. He, and Q. X. Yan, "The current situation and prospect of seismic analysis methods for tunnel structure," China Civil Engineering Journal, vol. 46, no. S1, pp. 262-268, 2013.

[2] Y. S. Shen, L. Z. Tang, and P. F. Zhou, "Research on antiseismic technology of railway tunnel structure crossing soft and hard interface in strong earthquake area," Railway Standard Design, vol. 62, no. 10, pp. 123-129, 2018.

[3] L. M. Qu, X. M. Ding, G. Kouroussis, and C. J. Zheng, "Dynamic interaction of soil and end-bearing piles in sloping ground: numerical simulation and analytical solution," Computer and Geotechnics, vol. 134, 2021.

[4] J. M. Zhou, Y. Cheng, S. W. Zou, Y. Zhou, and K. Huang, "Research on tunnel mechanical characteristics under fault movement and earthquake," Railway Standard Design, vol. 63, no. 11, pp. 138-144, 2019.

[5] Y. Zhang, Research on Damage and Cracking of Tunnel Lining under Fault Creeping and Fault Action and Flexible Connection Anti-fault Measures, Southwest Jiaotong University, Chengdu, China, 2016.

[6] Y. Zhao, E. D. Guo, Z. Liu, and L. Gao, "Damage analysis of urban metro tunnel under strike-slip fault," Rock and Soil Mechanics, vol. 35, no. S2, pp. 467-473, 2014.

[7] Y. Zhao, Seismic Response Analysis of Subway Tunnel in Active Fault area, Institute of Engineering Mechanics, China Earthquake Administration, Harbin, China, 2014.

[8] P. Geng, Y. He, C. He, Q. L. Quan, and Q. X. Yan, "Study on the reasonable seismic fortification length of the tunnel crossing the fracture zone," Chinese Journal of Rock Mechanics and Engineering, vol. 33, no. 2, pp. 358-365, 2014.

[9] C. L. Xin, Research on the Mechanism of Ground Motion Damage and Anti-seismic Measures for Tunnels Crossing faults, Southwest Jiaotong University, Chengdu, China, 2015.

[10] D. S. Chen, "The empirical relationship between the length of the surface rupture caused by an earthquake and the magnitude of the earthquake," North China Earthquake Sciences, vol. 2, no. 2, pp. 26-32, 1984.

[11] D. L. Wells and K. J. Coppersmith, "New empirical relationships among magnitude, rupture length, rupture width, rupture area, and surface displacement," Bulletin of the 
Seismological Society of America, vol. 84, no. 4, pp. 974-1002, 1994.

[12] D. A. Cole and P. V. Lade, "Influence zones in alluvium over dip-slip faults," Journal of Geotechnical Engineering, vol. 110, no. 5, pp. 599-615, 1984.

[13] M. F. Bransby, M. C. R. Davies, A. El Nahas, and S. Nagaoka, "Centrifuge modelling of reverse fault-foundation interaction," Bulletin of Earthquake Engineering, vol. 6, no. 4, pp. 607-628, 2008.

[14] M. F. Bransby, M. C. R. Davies, and A. E. Nahas, "Centrifuge modelling of normal fault-foundation interaction," Bulletin of Earthquake Engineering, vol. 6, no. 4, pp. 585-605, 2008.

[15] M.-L. Lin, C.-F. Chung, F.-S. Jeng, and T.-C. Yao, “The deformation of overburden soil induced by thrust faulting and its impact on underground tunnels," Engineering Geology, vol. 92, no. 3/4, pp. 110-132, 2007.

[16] M. L. Lin, F.-S. Jeng, T. H. Huang et al., "A study on the damage degree of shield tunnels submerged in overburden soil during the thrust fault offset," in Proceedings of the PVP 2006 ASME Pressure Vessels and Piping Division Conference, Vancouver, BC, Canada, July, 2006.

[17] M. L. Lin and F. S. Jeng, "Response of soil and a submerged tunnel during a thrust fault offset based on model experiment and numerical analysis," in Proceedings of ASME Pressure Vessels and Piping Division Conference, Denver, CO, USA, July 2005.

[18] M.. Sabagh and A.. Ghalandarzadeh, "Numerical modelings of continuous shallow tunnels subject to reverse faulting and its verification through a centrifuge," Computers and Geotechnics, vol. 12, pp. 1873-7633, 2020.

[19] S. An, L. J. Tao, X. C. Han, and Y. Zhang, "Application of twolevel design method on subway tunnel crossing active fault: a case study on Urumqi subway tunnel intersected by reverse fault dislocation," Bulletin of Engineering Geology and the Environment, vol. 3, pp. 1435-9529, 2021.

[20] Z. Zhang, R. Gong, H. Zhang, Q. Lan, and X. Tang, "Initial ground stress field regression analysis and application in an extra-long tunnel in the western mountainous area of China," Bulletin of Engineering Geology and the Environment, 2021.

[21] Z. G. Tao, S. L. Ren, G. Li, H. T. Xu, S. L. Luo, and M. C. He, "Model test on support scheme for carbonaceous slate tunnel in high geostress zone at high depth," Journal of Mountain Science, vol. 3, no. 18, pp. 1672-6316, 2021.

[22] W. Zhang, Research on Seismic Response and Damping Measures of Large-Diameter Shield Tunnel Structure, Institute of Rock and Soil Mechanics, Chinese Academy of Sciences, Wuhan, China, 2009.

[23] Z. Y. Chen and Y. Zhao, "Analysis of tunnel response caused by active reverse fault movement," in Proceedings of the 2019 National Engineering Geology Annual Conference, pp. 113118, The Geological Society of China: Editorial Office of Journal of Engineering Geology, Beijing, China, 2019.

[24] GB 50010-2010, Code for Design of Concrete Structures, China Architecture \& Building Press, Beijing, China, 2015.

[25] H. Huo, Seismic Design and Analysis of Rectangular Undergroundstructures, pp. 23-28, School of Civil Engineering, Purdue University, West Lafayette, IN, USA, 2005.

[26] J. W. Xu, "On the main problems of strike-slip fault," Frontiers of Earth Science, vol. 2, no. 2, pp. 125-136, 1995.

[27] H. L. Song, C. H. Zhang, and G. H. Wang, Structural Geology, Geological Publishing House, Beijing, China, 2013.

[28] X. Z. Liu, X. L. Wang, and L. L. Lin, "Model experimental study on influence of normal fault with $60^{\circ}$ dip angle stick-slip movement on mountain tunnel," Chinese Civil Engineering Journal, vol. 47, no. 2, pp. 121-128, 2014.

[29] Q. Fang, W. Su, D. L. Zhang, and F. C. Yu, "Research on deformation characteristics of tunnel surrounding rock based on field monitoring data," Chinese Journal of Rock Mechanics and Engineering, vol. 35, no. 9, pp. 1884-1897, 2016.

[30] G. L. Liu, Research on Key Technologies and Analysis and Forecast Methods of Metro Tunnel Deformation Monitoring, Wuhan University, Wuhan, China, 2013.

[31] W. B. Ma and J. F. Chai, "Development status of disease detection, monitoring, evaluation and treatment technology of operating railway tunnel," Tunnel Construction, vol. 39, no. 10 , pp. $1553-1562,2019$. 